Published in final edited form as:

ACS Catal. 2019 June 7; 9: 4862-4866. doi:10.1021/acscatal.9b00851.

\title{
Cobalt-Catalyzed Alkylation of Drug-Like Molecules and Pharmaceuticals Using Heterocyclic Phosphonium Salts
}

\author{
Xuan Zhang, Andrew McNally \\ Department of Chemistry, Colorado State University, Fort Collins, Colorado 80523, United States
}

\begin{abstract}
Alkylated pyridines are common in pharmaceuticals, and metal catalysis is frequently used to prepare this motif via $\mathrm{Csp}^{2}-\mathrm{Csp}^{3}$ coupling processes. We present a cobalt-catalyzed coupling reaction between pyridine phosphonium salts and alkylzinc reagents that can be applied to complex drug-like fragments and for late-stage functionalization of pharmaceuticals. The reaction generally proceeds at room temperature, and 4-position pyridine $\mathrm{C}-\mathrm{H}$ bonds are the precursors in this strategy. Given the challenges in selectively installing (pseudo)halides in complex pyridines, this two-step process enables sets of molecules to be alkylated that would be challenging using traditional cross-coupling methods.
\end{abstract}

\section{Graphical Abstract:}

Cobalt-Catalyzed Coupling of Pyridine Phosphonium Salts with Alkyl Zinc Reagents<smiles>Brc1ccc(CN2CCN(Cc3ccccc3)CC2)cc1</smiles>

1) C-P Bond-

Formation

2) Co-Catalyzed

Coupling

Room temperature coupling

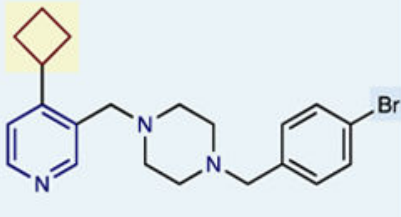

- Chemoselective

\section{Keywords}

pyridines; alkylation; late-stage; phosphonium salts; cross-coupling; cobalt-catalysis; alkyl Negishi

Pyridines are important pharmacophores in therapeutic compounds, but their precise function is a combined effect of the heterocycle and its adorning substituents. ${ }^{1}$ Alkylated pyridines are particularly common, and examples of their occurrence in marketed drugs are shown in eq 1 . The alkyl groups serve various roles in drug development, such as occupying hydrophobic pockets, changing binding properties of the Lewis basic nitrogen atom,

*Corresponding Author: andy.menally@colostate.edu.

Supporting Information

The Supporting Information is available free of charge on the ACS Publications website at DOI: 10.1021/acscatal.9b00851.

Experimental procedures and spectral data (PDF)

The authors declare no competing financial interest. 
protecting against oxidative metabolism as well as serving as linkers to other portions of the molecule. ${ }^{2}$ The effect of alkyl groups on pyridines is also relevant for other applications such as ligands, materials, and redox-active molecules in batteries. ${ }^{3,4}$ As such, methods to add alkyl groups to pyridines are broadly useful in several disciplines of applied chemistry.

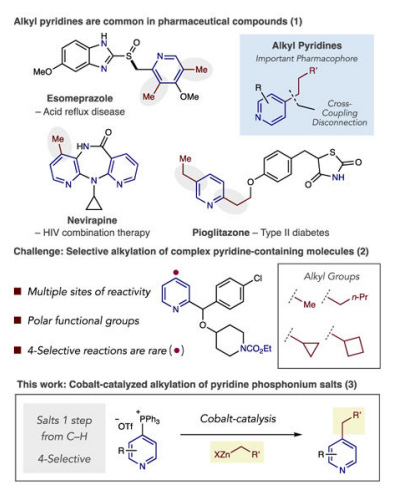

Coupling reactions are modular and efficient ways of forming $\mathrm{Csp}^{2}-\mathrm{Csp}^{3}$ bonds between pyridines and functionalized carbon-bearing groups. Several methods exist to install alkyl groups via $\mathrm{C}-\mathrm{H}$ functionalization reactions including Minisci-type reactions and metalcatalyzed coupling reactions with alkenes. ${ }^{5,6}$ Despite significant progress, controlling regioselectivity and tolerating a broad range of pyridines can be problematic in these respective reaction types. Adding organometallics to pyridinium salts is another approach, and Fier recently showed an example where an alkyl group was added via this reaction pathway. ${ }^{7}$ The most common methods to make alkylated pyridines are transition metalcatalyzed cross-coupling reactions between pyridyl(pseudo)halides and alkyl organometallic reagents. ${ }^{8}$ These reactions are broadly effective to alkylate pyridine building blocks where halide or pseudohalides are commercially available, or can be prepared. In drug development, however, pyridine-containing molecules are often complex and devoid of cross-coupling handles. Furthermore, these molecules have multiple reactive sites, substitutional variability and an excess of polar function groups making selective (pseudo)halogenation of $\mathrm{C}-\mathrm{H}$ bonds challenging, or impossible, using existing methods (eq 2). Our goal was therefore to address this challenge using an alternative cross-coupling precursor, and herein we present a cobalt-catalyzed coupling reaction between alkylzinc reagents and pyridine phosphonium salts (eq 3). The reaction operates at room temperature and forms a diverse set of alkylated pyridines from $\mathrm{C}-\mathrm{H}$ precursors.

Our laboratory is developing a program where phosphonium groups can serve as generic functional handles to functionalize pyridines and diazines. ${ }^{9}$ This strategy overcomes significant deficiencies of using heteroaryl (pseudo)halides as the $\mathrm{C}-{ }^{+} \mathrm{PPh}_{3}$ group can be directly and selectively installed on a broad range of substrates, and subsequent transformations are compatible with polar functional groups that are often found in drug-like molecules. ${ }^{10}$ Furthermore, the reaction is 4 -selective for pyridines, a position that is difficult to access using most methods. We have previously shown that phosphonium ions can serve as pseudohalides in a nickel-catalyzed coupling process with (hetero)aryl boronic acids. Combining a Ni(0) catalyst with an NHC ligand was crucial for selective heterocycle vs phenyl coupling using $\mathrm{PPh}_{3}$-derived azine salts. ${ }^{9 \mathrm{~d}}$ Our attempts at developing an alkylation 
reaction began with pyridine phosphonium salt $\mathbf{1 a}$ and butylzinc as a coupling partner in THF at $50{ }^{\circ} \mathrm{C}$ (Table 1). Entry 1 shows that our previous nickel system results in an unselective mixture of butylpyridine 2a and phenyl-coupling product $\mathbf{3}$. Using phosphine or bipyridine ligands were similarly unselective, as were ligated palladium catalysts (entries 25). We instead turned our attention to cobalt catalysis and tested a Co(III) salt with bipyridine (L1) as a ligand. ${ }^{11,12}$ Gratifyingly, the reaction was selective for pyridine 2a with only traces of butylbenzene 3 observed in the reaction mixture (entry 6), although the reaction efficiency was low. Conducting the reaction at room temperature resulted in a similar yield of 2a (entry 7). Methoxy substituted bipyridine $\mathbf{L} \mathbf{2}$ was significantly more effective as a ligand indicating that increased electron density at the metal center improves reactivity. A screen of Lewis basic additives revealed that $N$-Me imidazole further increase the efficiency of the reaction (entries 8 and 9). ${ }^{13}$ We hypothesized that the additive sequesters the ZnClOTf byproduct; entry 10 shows that adding a $\mathrm{Zn}$ (II) salt at the outset of the reaction is deleterious to the yield of the process and supports our hypothesis. The reaction was further improved by decreasing the concentration to $0.033 \mathrm{M}$ (entry 11), and employing cyclohexyloxy-substituted bipyridine $\mathbf{L 3}$ is the most effective protocol (entry 12). We assume a low valent ligated cobalt species $(\operatorname{Co}(0)$ or $\operatorname{Co}(\mathrm{I}))$ is the active catalyst and a typical oxidative addition-transmetalation-reductive elimination sequence constitutes the catalytic cycle.

The scope of alkylzinc reagents was examined using phosphonium salt $\mathbf{1 b}$ as a representative substrate (Table 2). ${ }^{9 \mathrm{f}}$ Linear zinc reagents, containing phenyl, chloro, cyano, and ester groups, provide products $\mathbf{2 b - 2 e}$ in moderate to good yields ( $\mathbf{2 b}-\mathbf{2} \mathbf{e})$; conducting the reaction at $50{ }^{\circ} \mathrm{C}$ is optimal in the latter two cases. Silyl ethers and carbazole fragments are also tolerated ( $\mathbf{2} \mathbf{f}$ and $\mathbf{2 g}$ ). Benzylzinc is a reasonable coupling partner (2h), and cyclopropyl- and cyclobutylzincs result in high yields of alkylated products ( $\mathbf{2} \mathbf{i}$ and $\mathbf{2 j}$ ). Cyclopentylzincs are less effective in this process, but usable quantities of $\mathbf{2 k}$ were obtained; we are uncertain of the exact reasons for the decreased yields, but we presume that an unfavorable steric interaction is operative. This point was exemplified when iso-propylzinc was examined; an isomeric mixture of products $\mathbf{2} \mathbf{I}$ and $\mathbf{2} \mathbf{m}$ were obtained with the linear product significantly favored. Our hypothesis is that branched to linear isomerism occurs at a $\mathrm{Co}$ (II)-species via a reversible $\beta$-hydride elimination-hydrometalation sequence and that the less-hindered linear alkylcobalt isomer undergoes reductive elimination more rapidly. ${ }^{14}$ Examples of unsuccessful coupling reactions include allyl zinc reagents, where a complex mixture of products was observed, $\boldsymbol{a}$-zinc carbonyls, and a iodozinc amino acid derivative.

In Table 3, we applied the phosphonium-mediated coupling process to a range azinecontaining structures. Pyridine building blocks containing functional groups such as cyano, trifluoromethyl, esters, methoxy, and boronic esters are tolerated (2n-2r). Quinoline salts are amenable to the strategy and $\mathbf{2 s}$ was obtained as a single regioisomer. A 3-fluoropyridine salt performed well in the Co-coupling reaction (2t); however, a 2-substituted isomer was formed along with the corresponding bis-alkylated product in a 3.5:1 ratio (2u). Substrates that result in low salt yields or give no C-P bond-formation include 2,6-disubstituted pyridines, acridines, $2-\mathrm{CF}_{3}$-pyridines, and pyridines with more than two electronwithdrawing groups or electron-donating groups. Limitation of pyridyl phosphonium salts in 
the zinc coupling process include chloro-, bromo-, and iodopyridines that result in mixtures of alkylated products via the $\mathrm{C}-\mathrm{Hal}$ and $\mathrm{C}-\mathrm{P}$ bonds as well as bis-alkylation (vide infra). At this point, 2-pyridylphosphonium salts are unsuccessful as coupling partners as well as salts derived from 2,2-bipyridines. Amino substituents at the 2-position lead to trace amounts of products and attempted alkylation of a 3,5-dimethylphonium salt resulted in return of the $\mathrm{C}-$ $\mathrm{H}$ precursor.

Next, a set of complex azines that approximate structures encountered in medicinal chemistry programs was tested. ${ }^{10}$ Starting with 3 -substituted pyridines, nicotine could be taken through the two-step process and alkylated in moderate yield (2w). Alkylated pyridines $2 \mathbf{x}$ and $2 \mathbf{y}$ are notable because of the presence of other heterocycles and basic amines. Site-selective alkylation reactions are desirable, and a butyl group could be selectively installed on the 3 -substituted pyridine in $\mathbf{2 z}$ given the preference of forming phosphonium salts on 3-substituted pyridines over 2-substituted isomers. Pyridinecontaining structures possessing a benzhydril center, a protected pyrrolidine (2aa and 2ab) as well as a precursor to the antihistamine bepotastine (2ac), can also be alkylated. Quinolines are alkylated at the 2-position when the 4-position is substituted (2ad and 2ae). A pyrimidine was also alkylated in this protocol, and although the yield of $2 \mathbf{a f}$ was low, a single regioisomer was formed; $\mathrm{C}-\mathrm{P}$ bond cleavage and return of the $\mathrm{C}-\mathrm{H}$ precursors was the main side product in this reaction. Methylation of azines is a common strategy in drug development, and we tested $\mathrm{MeZnCl}$ in this coupling process. ${ }^{15,16}$ While less efficient than $n-\mathrm{BuZnCl}$ as a coupling reagent, four examples of pyridines and quinolines were alkylated in reasonable yields (2ag-2aj). ${ }^{17}$

Late-stage functionalization of therapeutic compounds is an area of current importance in medicinal chemistry, and we examined five drug compounds in the phosphonium-mediated strategy. ${ }^{18}$ The antihistamine, chlorphenamine, is effective in this protocol with butylated and cyclobutylated derivatives 2ak and 2al obtained in good yields. Loratadine was cyclopropanated at the 4-position of the pyridine moiety in excellent yield (2am). Pyriproxyfen, a pesticide, is also alkylated efficiently (2an). A steroidal treatment for prostate cancer, abiraterone acetate, can be conveniently converted into alkylated derivative 2ao, and a protected version of varenicline, possessing a quinoxaline core, is alkylated adjacent to the heterocyclic nitrogen atom (2ap).

We next explored chemoselective Co-catalyzed couplings and site-selective switching reactions in polyazine substrates (Scheme 1). In our Ni-catalyzed Suzuki reaction, aryl bromides preferentially react over pyridylphosphonium salts. ${ }^{8 \mathrm{e}}$ In this Co-catalyzed process, we found the opposite order of chemoselectivity. Salt 1aq, containing an aryl bromide, was subjected to the standard coupling conditions at a short reaction time. The major outcome is cyclobutylation of the pyridine ring (2aq) with minor amounts of debrominated product 2ar. No evidence of coupling via the $\mathrm{C}-\mathrm{Br}$ bond was detected in the reaction mixture, unlike our observations of halopyridines. Using our previously developed method to control siteselective $\mathrm{C}-\mathrm{P}$ bond-formation, we made two phosphonium ion isomers of loratadine analogue 1as in $>20: 1$ selectivity. ${ }^{\text {h }}$ Each isomer was subsequently alkylated to make cyclopropane analogues 2as and 2at in reasonable yields and demonstrates the compatibility of the Co-coupling process with the site-selective switching strategy. 
In summary, we have shown that pyridine phosphonium salts, selectively installed in one step from $\mathrm{C}-\mathrm{H}$ precursors, can serve as coupling partners in a cobalt-catalyzed crosscoupling reaction with alkylzinc reagents. This simple, room-temperature process can generate alkylated analogues in a range of complex pyridine-containing molecules and serve as a strategy for late-stage alkylation of pharmaceuticals. The distinct scope compared with methods employing halogenated heterocycles as partners will provide new opportunities in drug-development programs.

\section{Supplementary Material}

Refer to Web version on PubMed Central for supplementary material.

\section{ACKNOWLEDGMENTS}

This work was supported by The National Institutes of Health (NIGMS) under award number R01 GM124094 and partial support from the Donors of the American Chemical Society Petroleum Research Fund (ACS PRF56878DNI1).

\section{REFERENCES}

(1). (a)Baumann M; Baxendale IR An Overview of the Synthetic Routes to the Best Selling Drugs Containing 6-Membered Heterocycles. Beilstein J. Org. Chem 2013, 9, 2265-2319. [PubMed: 24204439] (b)Vitaku E; Smith DT; Njardarson JT Analysis of the Structural Diversity, Substitution Patterns, and Frequency of Nitrogen Heterocycles among U.S. FDA Approved Pharmaceuticals. J. Med. Chem 2014, 57, 10257-10274. [PubMed: 25255204]

(2). Joule JA; Mills K Heterocyclic Chemistry, 4th ed; Blackwell: Malden, MA, 2000.(b)Grimmett MR Halogenation of Heterocycles 0.2. 6-Membered and 7-Membered Rings. Adv. Heterocycl. Chem 1993, 58, 271-329.

(3). Wurtz RP Chiral Dialkylaminopyridine Catalysts in Asymmetric Synthesis. Chem. Rev 2007, 107, 5570-5595. [PubMed: 18072804]

(4). (a)Sevov CS; Brooner REM; Chénard E; Assary RS; Moore JS; Rodríguez-López J; Sanford MS Evolutionary Design of Low Molecular Weight Organic Anolyte Materials for Applications in Nonaqueous Redox Flow Batteries. J. Am. Chem. Soc 2015, 137, 14465-14472. [PubMed: 26514666] (b)Sevov CS; Hickey DP; Cook ME; Robinson SG; Barnett S; Minteer SD; Sigman MS; Sanford MS Physical Organic Approach to Persistent, Cyclable, Low-Potential Electrolytes for Flow Battery Applications. J. Am. Chem. Soc 2017, 139, 2924-2927. [PubMed: 28219237]

(5). (a)Duncton MA Minisci Reactions: Versatile CH-Functionalizations for Medicinal Chemists. MedChemComm 2011, 2, 1135-1161.(b)Seiple IB; Su S; Rodriguez RA; Gianatassio R; Fujiwara Y; Sobel AL; Baran PS Direct C-H Arylation of Electron-Deficient Heterocycles with Arylboronic Acids. J. Am. Chem. Soc 2010, 132, 13194-13196. [PubMed: 20812741] (c)Jin J; MacMillan DW Alcohols as Alkylating Agents in Heteroarene C-H Functionalization. Nature 2015, 525, 87-90. [PubMed: 26308895] (d)DiRocco DA; Dykstra K; Krska S; Vachal P; Conway DV; Tudge M Late-Stage Functionalization of Biologically Active Heterocycles Through Photoredox Catalysis. Angew. Chem., Int. Ed 2014, 53, 4802-4806.(e)Gui J; Zhou Q; Pan C-M; Yabe Y; Burns AC; Collins MR; Ornelas MA; Ishihara Y; Baran PS C-H Methylation of Heteroarenes Inspired by Radical SAM Methyl Transferase. J. Am. Chem. Soc 2014, 136, 4853 4856. [PubMed: 24611732] For an example of reductive coupling of halopyridines with alkenes, see: Boyington, A. J.; Riu, M.-L. Y.; Jui, N. T. Anti-Markovnikov Hydroarylation of Unactivated Olefins via Pyridyl Radical Intermediates. J. Am. Chem. Soc. 2017, 139, 6582-6585.

(6). Murakami K; Yamada S; Kaneda T; Itami K C-H Functionalization of Azines. Chem. Rev 2017, 117, 9302-9332. [PubMed: 28445033] (b)Nakao Y Transition-Metal-Catalyzed C-H Functionalization for the Synthesis of Substituted Pyridines. Synthesis 2011, 2011, 3209-3219. 
(7). (a)Fier PS A Bifunctional Reagent Designed for the Mild, Nucleophilic Functionalization of Pyridines. J. Am. Chem. Soc 2017, 139, 9499-9502. [PubMed: 28677963] (b)Bull JA; Mousseau JJ; Pelletier G; Charette AB Synthesis of Pyridine and Dihydropyridine Derivatives by Regioand Stereoselective Addition to $N$-Activated Pyridines. Chem. Rev 2012, 112, 2642-2713. [PubMed: 22352938]

(8). For reviews and examples, see:(a)Haas D; Hammann JM; Greiner R; Knochel P Recent Developments in Negishi Cross-Coupling Reactions. ACS Catal. 2016, 6, 1540-1552.(b)Jana R; Pathak TP; Sigman MS Advances in Transition Metal (Pd,Ni,Fe)-Catalyzed Cross-Coupling Reactions Using Alkyl-Organometallics as Reaction Partners. Chem. Rev 2011, 111, 1417-1492. [PubMed: 21319862] (c)Thapa S; Kafle A; Gurung SK; Montoya A; Riedel P; Giri R LigandFree Copper-Catalyzed Negishi Coupling of Alkyl-, Aryl-, and Alkynylzinc Reagents with Heteroaryl Iodides. Angew. Chem., Int. Ed 2015, 54, 8236-8240.(d)Yang Y; Niedermann K; Han C; Buchwald SL Highly Selective Palladium-Catalyzed Cross-Coupling of Secondary Alkylzinc Reagents with Heteroaryl Halides. Org. Lett 2014, 16, 4638-4641. [PubMed: 25153332] (e)Hansen EC; Li C; Yang S; Pedro D; Weix DJ Coupling of Challenging Heteroaryl Halides with Alkyl Halides via Nickel-Catalyzed Cross-Electrophile Coupling. J. Org. Chem 2017, 82, 7085-7092. [PubMed: 28682073] (f)Li H; Zhong Y-L; Chen C-Y; Ferraro AE; Wang D A Concise and Atom-Economical Suzuki-Miyaura Coupling Reaction Using Unactivated Trialkyland Triarylboranes with Aryl Halides. Org. Lett 2015, 17, 3616-3619. [PubMed: 26125106] (e)Dreher SD; Dormer PG; Sandrock DL; Molander GA Efficient Cross-Coupling of Secondary Alkyltrifluoroborates with Aryl Chlorides-Reaction Discovery Using Parallel Microscale Experimentation. J. Am. Chem. Soc 2008, 130, 9257-9259. [PubMed: 18582050] (g)Malhotra S; Seng PS; Koenig SG; Deese AJ; Ford KA Chemoselective $s p^{2}-s p^{3}$ Cross-Couplings: IronCatalyzed Alkyl Transfer to Dihaloaromatics. Org. Lett 2013, 15, 3698-3701. [PubMed: 23829418]

(9). (a)Anders E; Markus F Neue Methode Zur Regiospexifischen Substitution Einiger Reaktionstracer N-Heteroaromatischer Ring-systeme. Tetrahedron Lett. 1987, 28, 2675-2676.(b)Anders E; Markus F Chemie der Triphenyl-(oder Tri-n-butyl-)-pyridylphosphoniumsalze, 1 Neue Methode zur Regioselektiven Einführung von Phosphoniumgruppen in $N$-heteroaromatische Ringsysteme. Chem. Ber 1989, 122, 113-118.(c)Hilton MC; Dolewski RD; McNally A Selective Functionalization of Pyridines via Heterocyclic Phosphonium Salts. J. Am. Chem. Soc 2016, 138, 13806-13809. [PubMed: 27731999] (d)Zhang X; McNally A Phosphonium Salts as Pseudohalides: Regioselective Nickel-Catalyzed Cross-Coupling of Complex Pyridines and Diazines. Angew. Chem., Int. Ed 2017, 56, 9833-9836.(e)Anderson RG; Jett BM; McNally A Selective Formation of Heteroaryl Thioethers via a Phosphonium Ion Coupling Reaction. Tetrahedron 2018, 74, 3129-3136. [PubMed: 30479455] (f)Koniarczyk JL; Hesk D; Overgard A; Davies IW; McNally A A General Strategy for Site-Selective Incorporation of Deuterium and Tritium into Pyridines, Diazines, and Pharmaceuticals. J. Am. Chem. Soc 2018, 140, 1990-1993. [PubMed: 29377684] (g)Patel C; Mohnike M; Hilton MC; McNally A A Strategy to Aminate Pyridines, Diazines, and Pharmaceuticals via Heterocyclic Phosphonium Salts. Org. Lett 2018, 20, 2607-2610. [PubMed: 29664307] (h)Dolewski RD; Fricke PJ; McNally A Site-Selective Switching Strategies to Functionalize Polyazines. J. Am. Chem. Soc 2018, 140, 8020-8026. [PubMed: 29792698] (i)Dolewski RD; Hilton MC; McNally A 4-Selective Pyridine Functionalization Reactions via Heterocyclic Phosphonium Salts. Synlett 2018, 29, 8-14.

(10). Blakemore DC; Castro L; Churcher I; Rees DC; Thomas AW; Wilson DM; Wood A Organic Synthesis Provides Opportunities to Transform Drug Discovery. Nat. Chem 2018, 10, 383-394. [PubMed: 29568051]

(11). For reviews on cobalt-catalyzed cross-coupling reactions, see:(a)Hammann JM; Hofmayer MS; Lutter FH; Thomas L; Knochel P Recent Advances in Cobalt-Catalyzed Csp $p^{2}$ and Csp $p^{3}$ CrossCouplings. Synthesis 2017, 49, 3887-3894.(b)Cahiez G; Moyeux A Cobalt-Catalyzed CrossCoupling Reactions. Chem. Rev 2010, 110, 1435-1462. [PubMed: 20148539] (c)Gosmini G; Bégouin J-M; Moncomble A Cobalt-Catalyzed Cross-Coupling Reactions. Chem. Commun 2008, 3221-3233.

(12). Cobalt halides can be used as catalysts but are marginally less efficient than $\mathrm{Co}(\mathrm{acac})_{3}$. See Supporting Information for more details. 
(13). See the Supporting Information for an additive screen and evaluation of other solvents (toluene, 1,4-dioxane, $\mathrm{CH}_{2} \mathrm{Cl}_{2}$, and $\mathrm{MeCN}$ ).

(14). O’Neill M; Cornella J Retaining Alkyl Nucleophile Regiofidelity in Transition-Metal-Mediated Cross-Couplings to Aryl Electrophiles. Synthesis 2018, 50, 3974-3996.

(15). Schönherr H; Cernak T Profound Methyl Effects in Drug Discovery and a Call for New C-H Methylation Reactions. Angew. Chem., Int. Ed 2013, 52, 12256-12267.

(16). Ritter recently reported a Ni-catalyzed methylation of pharmaceutically-relevant molecules via ammonium salts as precursors, see:Serpier F; Pan F; Ham WS; Jacq J; Genicot C; Ritter T Selective Methylation of Arenes: A Radical C-H Functionalization/Cross-Coupling Sequence. Angew. Chem., Int. Ed 2018, 57, 10697-10701.

(17). In these cases, we found that adding a catalytic amount of $i$-PrMgCl to the reaction mixture improved the yields of methylated products. See Supporting Information for details.

(18). Cernak T; Dykstra KD; Tyagarajan S; Vachal P; Krska SW The Medicinal Chemist's Toolbox for Late Stage Functionalization of Drug-Like Molecules. Chem. Soc. Rev 2016, 45, 546-576. [PubMed: 26507237] 

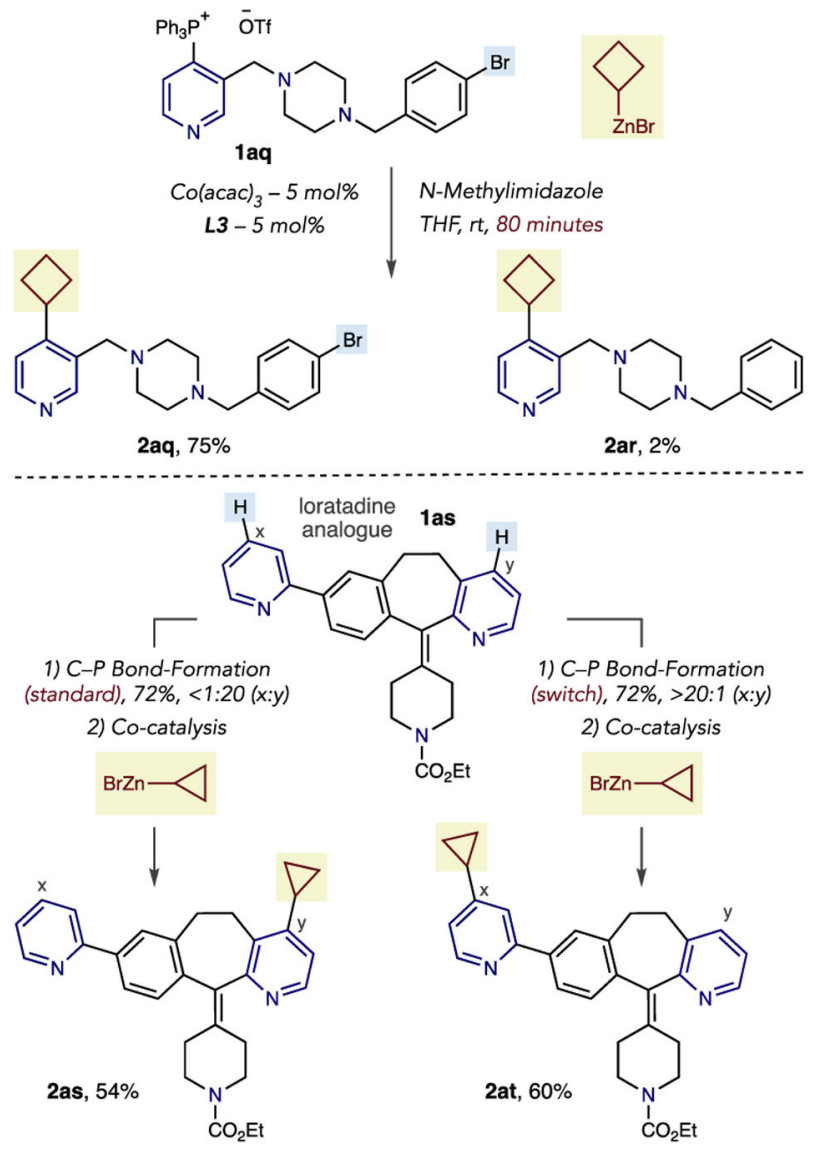

Scheme 1. Chemoselective Co-Catalyzed Alkylations ${ }^{a}$

${ }^{a}$ Isolated yields shown are of a mixture of 2aq and 2ar. Standard C-P bond formation: 1as (1.0 equiv), $\mathrm{Tf}_{2} \mathrm{O}$ (1.0 equiv), $\mathrm{PPh}_{3}$ (1.1 equiv), $\mathrm{DBU}$ (1.0 equiv) $\mathrm{CH}_{2} \mathrm{Cl}_{2},-78{ }^{\circ} \mathrm{C}$ to $\mathrm{rt}$.

Switch C-P bond formation: 1 as (1.0 equiv), $\mathrm{Tf}_{2} \mathrm{O}$ (2.0 equiv), $\mathrm{PPh}_{3}$ (2.0 equiv), $\mathrm{NEt}_{3}$ (2.0 equiv) $\mathrm{CH}_{2} \mathrm{Cl}_{2},-78{ }^{\circ} \mathrm{C}$ to rt. Co-catalysis: $10 \mathrm{~mol} \% \mathrm{Co}(\mathrm{acac})_{3}, 10 \mathrm{~mol} \% \mathbf{L 3}, \mathrm{N}$ Methylimidazole (1.5 equiv), THF, $50{ }^{\circ} \mathrm{C}$. 
Table 2.

Scope of Alkyl Zinc Reagents ${ }^{a}$

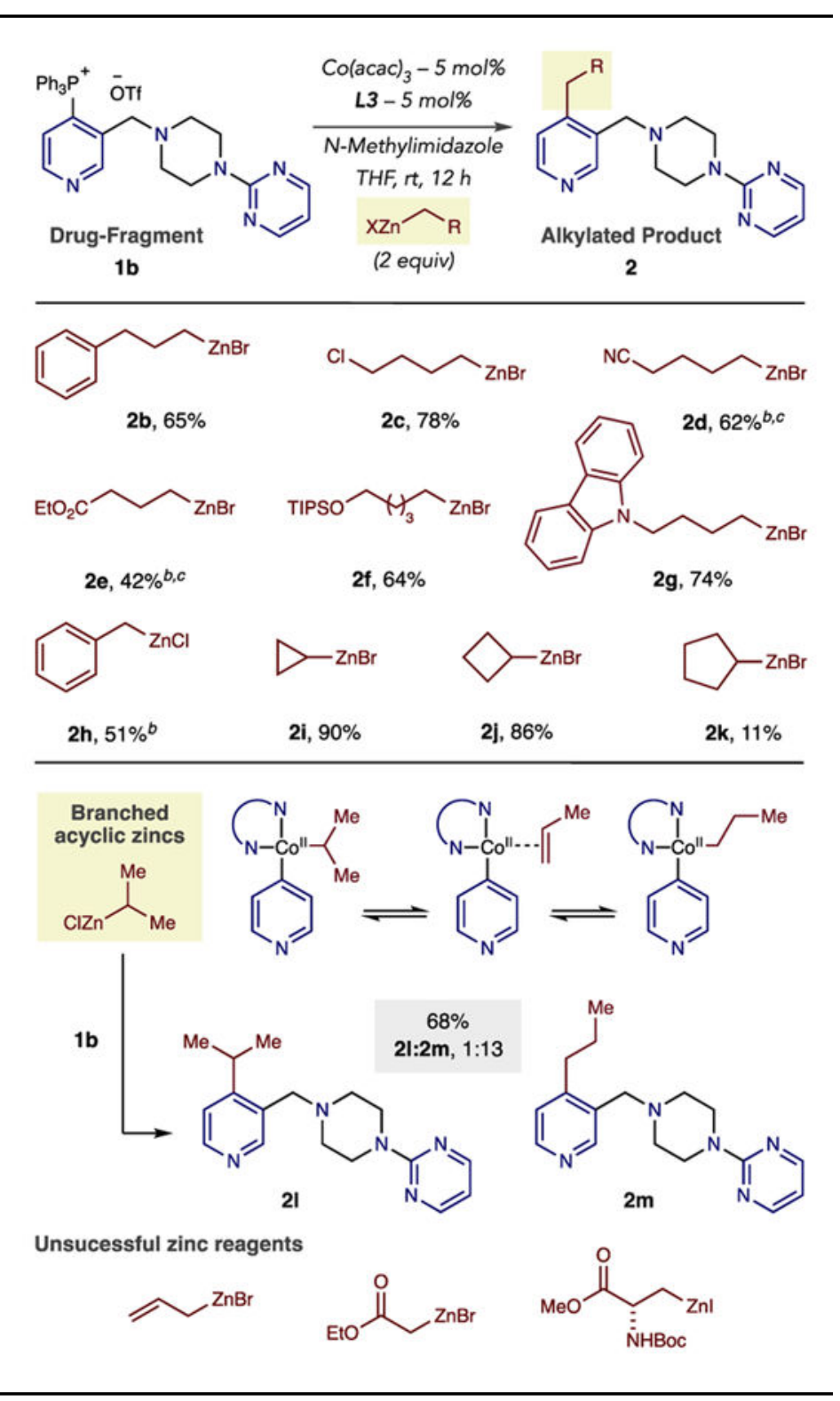

${ }^{a}$ Isolated yields of products are shown.

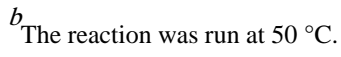

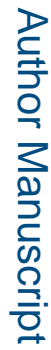

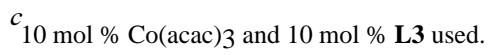



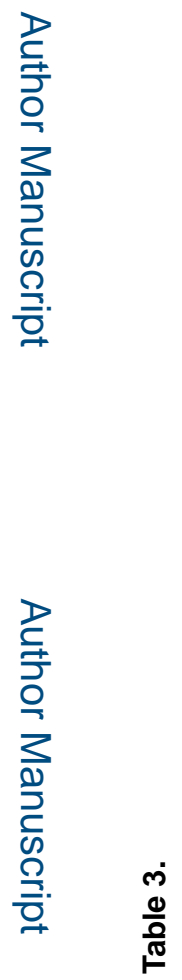

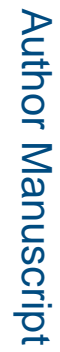

롤

ACS Catal. Author manuscript; available in PMC 2019 October 25. 


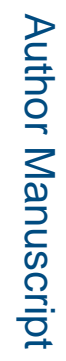

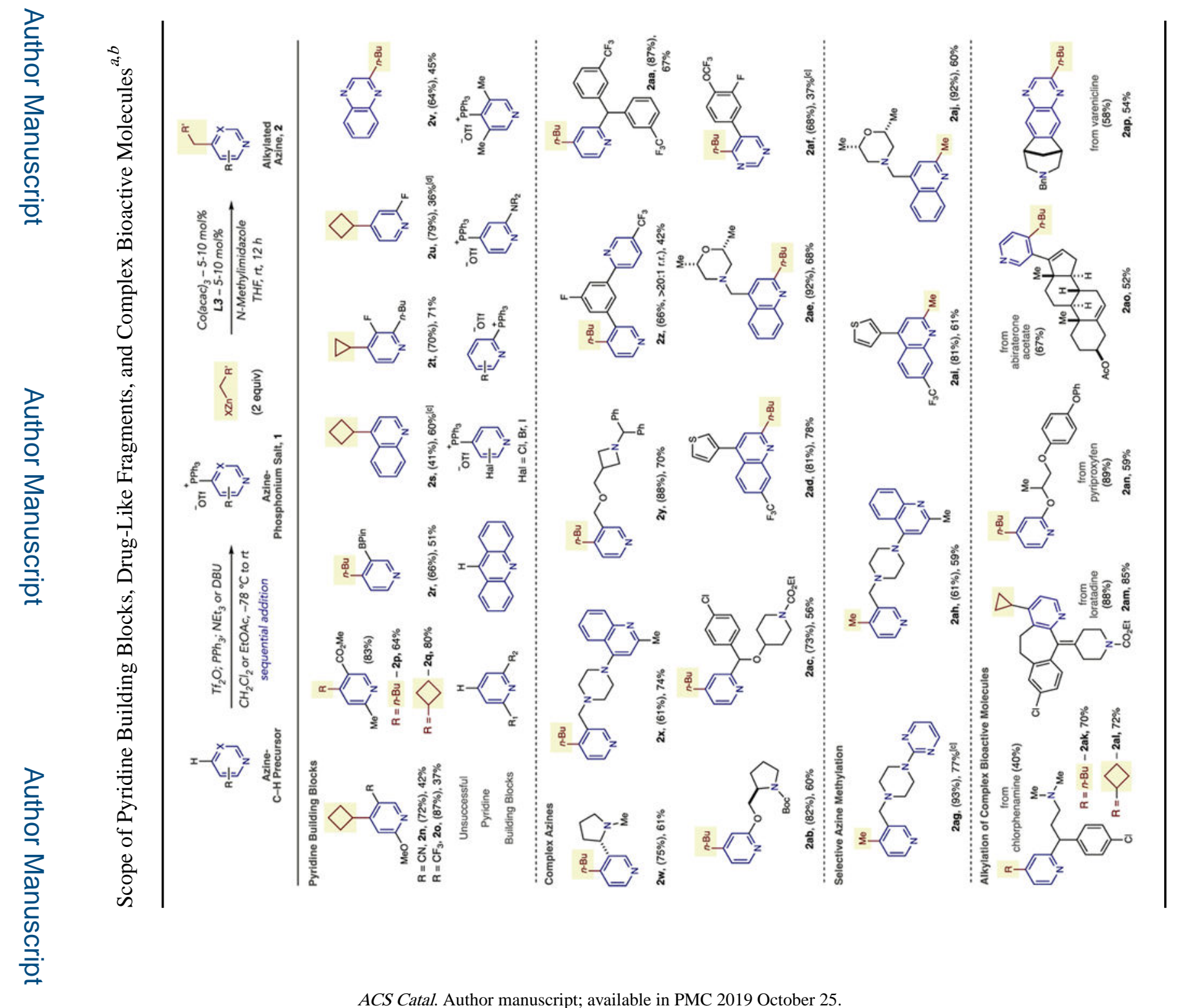



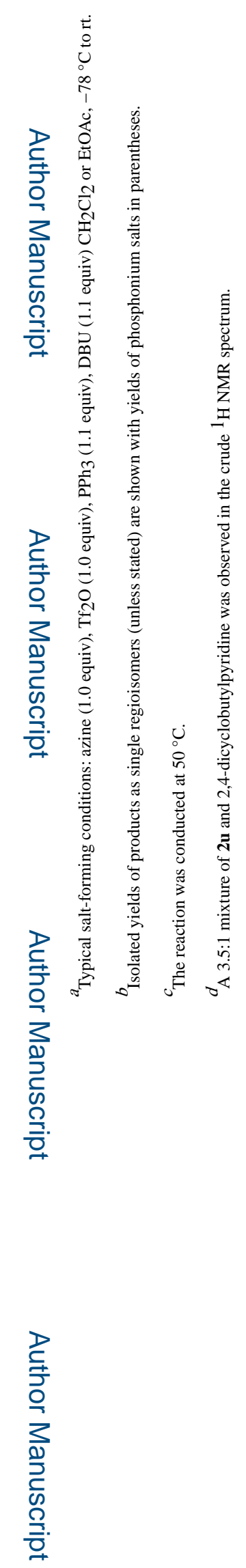

ACS Catal. Author manuscript; available in PMC 2019 October 25. 